\title{
Metacognitive judgements and abilities in patients with affective disorders
}

\author{
Barbara Drueke $^{1} \cdot$ Siegfried Gauggel ${ }^{1} \cdot$ Lorenz Weise $^{1} \cdot$ Thomas Forkmann $^{2} \cdot$ Verena Mainz $^{1}$ (i)
}

Accepted: 28 January 2022

(c) The Author(s) 2022

\begin{abstract}
Objectives Cognitive and metacognitive deficits depict important factors in depression, but the relationship between these concepts remains to be elucidated. The present study investigated the difference between patients with depression and controls in metacognitive judgements regarding the domain of attention. Furthermore, the associations between different metacognitive abilities, depressiveness and confidence were investigated, as well as in how far the derived correlates would predict depression.

Methods Thirty patients with a major depressive episode and 30 healthy participants were enrolled in the current study. Attention and executive functioning ability were assessed including metacognitive judgements of performance and confidence with regard to the test performance in the Stroop test. To examine further aspects related to (meta-)cognitive abilities, decentering skills, aspects of self-conscious attention, self-assessed intelligence and metacognitive beliefs, judgements and monitoring tendencies were assessed.

Results Albeit groups' metacognitive judgements of performance did not differ, patients indicated to be significantly less confident in their judgements. Depressive patients showed less decentering abilities compared to healthy participants and there was a significant association between decentering and confidence ratings. Moreover, depressiveness was associated with dysfunctional self-consciousness and low cognitive confidence. Finally, lower decentering skills and higher dysfunctional self-attention were the best predictors for depressiveness.

Conclusions Results favor the assumption that patients' metacognitive abilities regarding the domain of attention are not generally deficient. Rather, the lower confidence in their judgements and dysfunctional (meta-)cognitive abilities, like decentering, metacognitive beliefs and aspects of self-conscious attention and intelligence, seem to mirror the patients' impairments.
\end{abstract}

Keywords Metacognition $\cdot$ Decentering $\cdot$ Depression $\cdot$ Judgment of performance $\cdot$ Confidence

\section{Introduction}

A large number of studies have shown that different cognitive functions can be impaired to varying degrees in depressive patients (Marvel \& Paradiso, 2004; McClintock, Hussain, Greer, \& Cullum, 2010; Vasic, Wolf, \& Walter, 2007). A metaanalysis from Rock et al. (2014) reveals significant deficits in attention as well as in executive functions in

Verena Mainz

vmainz@ukaachen.de

1 Institute of Medical Psychology and Medical Sociology, University Hospital of the RWTH Aachen, Pauwelsstr. 19 (MTZ), 52074 Aachen, Germany

2 Department of Clinical Psychology, University of Duisburg-Essen, 45141 Essen, Germany patients with major depression, for example. Other studies have shown that depressive patients do not only have certain cognitive impairment, but also deficits in metacognitive processes, which are involved in the appraisal, monitoring, and control of cognitions (Flavell, 1979).

Support about the linkage between deficient metacognition and cognitive processes came from Papageorgiou and Wells (2001) whom were among the first to postulate metacognitive deficits in patients with depression. The authors assumed that metacognitive beliefs ('metacognitions') are an important vulnerability factor for maladaptive ruminations, i.e. somehow affecting cognitive (dys-)function in patients. Similarly, Sheppard and Teasdale (2004) found evidence for deficits in the metacognitive monitoring in patients.

Models of mental disorders, like the self-regulatory executive functioning model (S-REF, Wells \& Matthews, 
1996) or the more recent metacognitive model of depression (Wells, 2008), assumed both, cognitive processes like attention, and metacognition to be intermingled in pathology. The S-REF model integrates information processing research into Beck's schema theory by proposing attention capacity and reduced cognitive flexibility as important factors in metacognition when regarding emotional disorders (Wells $\&$ Matthews, 1996). The metacognitive model of depression stresses, that depression is mainly being maintained by rumination, which again is maintained by dysfunctional metacognitions (Wells, 2008). Recent studies supported this model at least in parts (Kraft et al., 2017; Aldahadha, 2021). For example, Kraft et al. (2017) found dysfunctional metacognitive beliefs in a mixed clinical-, non-clinical population to be associated with decreased executive control, and, negative metacognitions were associated with reduced ability to shift between mental sets. Similarly, Spada et al. (2010) described that metacognition about worry was related to self-reported decreased ability to shift and focus attention. However, in how far metacognition and the cognitive domain of attention, cognitive flexibility and executive functioning are empirically related has not yet been studied systematically.

To understand how metacognitive abilities could affect cognitive functions, especially attention processes or executive functioning, it is essential to look at metacognitive awareness as a related higher-level construct (Fresco et al., 2007; Hargus, Crane, Barnhofer, \& Williams, 2010; Teasdale et al., 2002). Within metacognition, the investigation of people's ability to monitor their own performance is a central topic, and monitoring as a metacognitive process refers to the subjective assessment of one's own cognitions and knowledge (Koriat \& Shitzer-Reichert, 2002). Metacognitive monitoring has mostly been studied in the field of memory and learning where it is typically assessed by means of metacognitive judgements (for a review see Koriat, 2007; Koriat \& Helstrup, 2007). More specifically, participants are asked to judge their own performance (judgement of performance, JOP) and afterwards, these judgements are compared to participants' real task performance. Furthermore, participants are asked how confident they feel in doing a certain task (judgement of confidence, JOC; cf. Koriat, 1997, 2007). A review by Hoven et al. (2019) summarized research regarding abnormal confidence judgements as a metacognitive operation reflecting a possible outcome of metacognitive monitoring as a 'subjective feeling of being correct about a choice, decision or statement' (Hoven et al., 2019, p. 1). Research revealed associations between various domains with regard to which confidence was affected through depression as well as other symptomatology (Hoven et al., 2019). Interestingly, associations between low confidence and depressiveness have been reported in studies that induced negative mood in healthy participants (Culot et al., 2021), and both, in studies investigating confidence judgements in samples with subclinical depressiveness (Moses-Payne et al., 2019; Rouault et al., 2018) as well as in clinical samples with major depression (Fu et al., 2005). These findings allow to take a rather general, transdiagnostic approach of understanding human metacognition as a quite basal cognitive ability or prerequisite for healthy functioning (e.g., Rouault et al., 2018). However, especially in the investigated subclinical samples findings remained partly inconclusive and associations between depressiveness and lower confidence seemed to depend on differences in performance accuracy, on whether the judgements of performance were indicated pro- or retrospectively and on the domain that was to be judged (Dunn et al., 2007; Hoven et al., 2019; Moses-Payne et al., 2019; Rouault et al., 2018).

Ehrlinger and Dunning (2003) were able to show that test performance and performance estimates were influenced by other factors too, like the participants' self-view. While there was no significant difference in test performance between 'high-esteem' and 'low-esteem' participants, their JOCs differed significantly. A study by Fu et al. (2012) revealed even lower retrospective confidence in performance in individuals with depressive symptoms as compared to healthy controls. In this study it is of note, that participants were able to build their judgement upon their actual performance, i.e. it was guided by actual knowledge. However, if you assess a confidence judgement before starting a specific task, you have to build your JOC on your rather basic believe about your own abilities in the test domain.

In how far prospective metacognitive confidence judgements differ between patients with depression and healthy controls has not yet been investigated and was, besides the assessment of the JOPs and the metacognitive accuracy in healthy and depressed participants, one aim of the present study. If metacognition is understood as the ability to make more or less reliable and valid judgements concerning one's own mental experience and behavior (Flavell, 1979) the metacognitive judgements should promote accurate selfassessments. Concerning patients with depression, there is evidence for the 'depressive realism hypothesis' which posits depressed people to have a more accurate view of reality, leading to more accurate self-assessments. Likewise, Soderstrom, Davalos, and Vázquez (2011) found mild depression to be associated with metacognitive accuracy. However, the same result could not be found in individuals suffering from moderate depression, and, there is also evidence for metacognitive dysfunction (e.g. Dunn et al., 2007; Fu et al., 2005; Kalska et al., 1999). For example, research revealed that depressive patients tend to underestimate their actual performance compared to controls (e.g. Fu et al., 2005; Kalska et al., 1999), and, that even when a positive bias was present in dysphoric and depressed participants too, compared to healthy controls, the extend of this bias was reduced and 
partly depended on whether the judgements concerned error or correct trials (Dunn et al., 2007).

Despite judgement accuracy, which can be assessed calculating the difference between the actual performance and judgements of performance and confidence, research describes further impacted metacognitive abilities in patients with affective disorders, which regard other, rather internally retrieved metacognitive dimensions, like for e.g. metamemory knowledge (Tournier \& Postal, 2011). Further, different assessment techniques are applied to assess metacognition, as for e.g. assessment of personal beliefs regarding (meta-) cognitive abilities using questionnaire surveys (e.g., Cipolli et al., 1996). With regard to these metacognitive abilities, metacognitive knowledge and metacognitive skills can be distinguished, that might be specifically related to cognitive processes of attention. One metacognitive skill, typically assessed via subjective indications is decentering (Nelson, 1990; Wells, 2000). Decentering describes a 'process through which one is able to step outside of one's immediate experience, thereby changing the very nature of that experience' (Safran \& Segal, 1990, p. 117). This enables a person to observe one's own mental processes from a distanced perspective by stepping outside from these. It implies a change in conscious processing, i.e. a shift away from the content of mental events towards a non-judgemental awareness, and even more, to the acceptance of them. With regard to decentering abilities, it has been shown that depressive patients had lower decentering abilities than healthy controls (Teasdale et al., 2002) and that decentering abilities were negatively correlated with relapse rates after therapy (Fresco et al., 2007; Teasdale et al., 2002). However, the conceptualization of decentering as a metacognitive strategy (Bernstein et al., 2015; Lebois et al., 2015) demanded an answer of its relationship to other cognitive and mental processes. It can be assumed that "decentering" as a metacognitive process is closely related to intellectual performance, since metacognitions through their control and monitoring function only enable cognitive processes such as the selection of adaptive behaviors (e.g. promising learning strategies) to be carried out (Borkowski, 1985; Sternberg, 1984).

The relationship between metacognitive abilities and cognitive functioning in patients with depression is an interesting question, but needed further research. Especially, the question of whether and how patients' metacognitive knowledge, skills and abilities are linked to the domain of attention was not clear yet. The study at hand aimed at investigating the interrelationship between cognitive and metacognitive processes in patients with depression and healthy controls. Cognitive functioning, specifically attention and executive performance, as well as the metacognitive abilities associated with cognitive monitoring, i.e. metacognitive judgements of performance and prospective judgements of confidence were investigated using the Stroop task, named Color
Word Interference Test (CWIT; Stroop, 1935). Both, a lower performance in the cognitive task and lower metacognitive monitoring abilities in depressive patients as compared to healthy controls were assumed. Additionally, metacognitive abilities addressing different internal metacognitive monitoring processes like decentering, self-assessed intelligence, self-conscious attention, cognitive confidence and self-consciousness are assumed to play a key role in cognitive functioning. A detailed investigation of the associations among these metacognitive measures and judgements of confidence were further aimed at, as well as to examine in how far the different correlates would predict depressiveness.

\section{Methods}

\section{Participants}

Thirty patients who met the criteria for a current depressive episode ( $60 \%$ female, $M_{\text {age }}=37.6, \mathrm{sd}=12.4$ ) were recruited shortly after admission to a psychiatric department at a university hospital. The patients' clinical diagnoses were completed by experienced psychiatrists and psychologists (see Table 1). Gender and age-matched healthy control participants were enrolled via advertisements $\left(\mathrm{M}_{\mathrm{age}}=38.2\right.$, sd $=12.4$ ). Exclusion criteria for the healthy participants were any physical or mental constraints orally reported during recruitment. Before the examination a telephone interview was conducted to acquire general demographic information. This was necessary to check for participation suitability of the control participants. The study was approved by the local ethics committee, and all participants provided written informed consent and received financial compensation for their participation.

\section{Procedure}

At the day of the examination all participants were informed about the experimental procedure. After agreement to the study all participants were interviewed with help of the

Table 1 Distribution of diagnoses in patient sample

\begin{tabular}{llll}
\hline Diagnosis ICD-10 & Severity & $N(30)$ & $\%$ \\
\hline Depressive episode & mild & 2 & 7 \\
& moderate & 2 & 7 \\
& severe & 1 & 3 \\
Recurrent depressive disorder, & mild & 4 & 13 \\
current episode ... & moderate & 14 & 47 \\
& severe & 7 & 23 \\
\hline
\end{tabular}

a acquired with the International Diagnose Checklist (ICDL; Hiller et al., 1996) 
International Diagnose Checklist for DSM-IV and ICD-10 (IDCL; Hiller, Zaudig, \& Mombour, 1996) to validate diagnoses. The IDCL is a clinical interview aimed at reaching a precise diagnosis according to DSM-IV and ICD-10 classification. It was conducted by a research assistant who was trained and clinically experienced. Furthermore, participants filled out a sociodemographic questionnaire and performed an assessment of intelligence. Intelligence was assessed using the Wortschatztest (WST; Schmidt \& Metzler, 1992) which enables a quick assessment of the participants' verbal intelligence level and the evaluation of the speech comprehension. The WST offers the opportunity to estimate the intelligence of the participants using norms and IQ values for the age between 20 and 90 . The groups did not differ significantly concerning age or IQ. Next, they filled out the Rasch-based depression screening (DESC, Forkmann et al., $2009,2010)$ to acquire measures of clinical and subclinical depression severity. Higher scores indicate more symptoms of depression and total scores of $\geq 12$ suggests the presence of a depressive episode (Forkmann et al., 2009). Cronbach's $\alpha$ for the DESC in the present sample was 0.9 indicating excellent internal consistency (Howitt \& Cramer, 2005). After, participants filled out all relevant measures with regard to the study hypotheses. Questionnaires or specific subscales thereof were collected to examine metacognitive abilities, aspects of self-conscious attention and individual estimations with regard to cognitive performance. Finally, attention and executive functioning ability were assessed including metacognitive judgements of performance and confidence with regard to the test performance. All assessments are described in detail below.

\section{Assessments}

\section{Decentering}

As a self-report measure for metacognitive decentering abilities participants filled out the German version of the Experience Questionnaire (EQ-D; Gecht et al., 2014), which was originally developed by Fresco et al. (2007). The EQ-D requires answering 20 items on whether one has recently had "similar experiences" (e.g., "I view things from a wider perspective") using a 5-point Likert scale (ranging from 'never' (0) to 'all the time' (4)). Psychometric analyses of the EQ-D revealed adequate internal consistency and construct validity, suggesting basing data analyses and the interpretation on the 8-item solution derived from the psychometric evaluation of the German version. Interpretation of decentering scores focuses on the two first-order factors "Accepting Self-Perception" (Factor 1: EQ-ASP) and "Distanced Perspective" (Factor 2: EQ-DP). The first factor, EQ-ASP, consists of four items and comprises appreciating oneself and refers to a state with regard to how people experience themselves as a person, being aware that one's own characteristics belong and originate from oneself. The second factor, EQ-DP, consists of four items and refers to the cognitive ability to have a reflective and observing perspective on one's own inner experiences and mental processes. Per subscale total scores can be calculated that can range from 0 to 16 with higher scores indicating higher decentering abilities. In the present sample, Cronbach's $\alpha$ of both subscales was 0.8 indicating high internal consistency (Howitt \& Cramer, 2005).

\section{Self-conscious Attention}

Functional and dysfunctional aspects of self-conscious attention were examined with the Functional and Dysfunctional Self-consciousness questionnaire (DFS; Hoyer, 2000). The instrument allows distinguishing two scales and involves answering 22 items on a 5-point Likert-scale (ranging from $1=$ 'not at all applicable' to $5=$ 'totally applicable'). The first scale, "dysfunctional self-consciousness" (DFS-DS) measures an inflexible or prolonged state of self-focused attention as characteristic for dysfunctional cognitive operations which lack flexibility (e.g. "I think about my mistakes for a long time, even if there is nothing I can do about it."). The second scale, "functional self-consciousness" (DFS-FS) describes a more flexible and adaptive self-focused attention. It represents regulative aspects of self-consciousness such as the recognition of behavioral borders and the generation of effective alternatives, thereby involving aspects of confidence in problem-solving abilities (e.g. "Provided I have enough time and am stuck behind it, I can solve most of my problems."). Higher values indicate higher functional or dysfunctional aspects of attention, respectively. Cronbach's $\alpha$ for DFS-DS in the present sample was 0.9, indicating excellent internal consistency. Cronbach's $\alpha$ for DFS-FS was 0.8 , indicating high internal consistency (Howitt \& Cramer, 2005).

\section{Self-assessed Intelligence}

The Inventar zur Selbsteingeschätzten Intelligenz (ISI; Rammstedt \& Rammsayer, 2002) is an inventory measuring self-assessed intelligence. The ISI consists of 11 items, each of which describes different aspects of intelligence. Participants have to estimate the extent of the perceived intelligence on $90 \mathrm{~mm}$ long visual analogue scales ranging from -3 to +3 . The resulting five ranges of the scale are marked as "extremely low intelligence" (-3 to -2$)$, "low intelligence" (-2 to -1$)$, "average intelligence" ( -1 to +1$)$, "high intelligence" $(+1$ to +2$)$ and "extremely high intelligence" $(+2$ to +3$)$. To calculate the self-assessed intelligence in a certain area, the value marked on the corresponding analogue scale is measured. Since the centre of the $90 \mathrm{~mm}$ long scales corresponds to an average IQ value of 100 IQ points, the amount 55 is 
added to the self-assessments in millimeters to convert them into IQ equivalents. The scale thus reflects self-assessments between 55 and 145 IQ points. The present study focuses on two scales of rather classical intelligence areas namely selfestimated verbal (VI) and mathematical-logical intelligence (MLI) as these aspects relate best to the demands and performance in the subsequent tasks (judgements of performance and confidence). Cronbach's $\alpha$ for VI in the present sample was 0.8 , indicating high internal consistency. Cronbach's $\alpha$ for MLI was 0.7 , indicating satisfactory internal consistency (Howitt \& Cramer, 2005).

\section{Metacognition}

The short form of the Metacognitions Questionnaire (MKF30; Wells \& Cartwright-Hatton, 2004; Arndt et al., 2011) is a brief multidimensional measure of metacognitions, measuring metacognitive beliefs, judgements and monitoring tendencies. Participants rate their agreement to 30 individual statements on a 4-point Likert-scale (ranging from $1=$ 'do not agree' to $4=$ 'agree very much'). The instrument has five subscales, which represent cognitive confidence, positive beliefs about worry, cognitive self-consciousness, negative beliefs about uncontrollability of thoughts and danger, and beliefs about need to control thoughts. In the study at hand, again, we focus on the two most relevant subscales with regard to our research questions: first, the "cognitive confidence" scale (CC) which contains items that refer to thoughts about the effectiveness of one's own cognitive abilities, especially memory and attention and, second, the "cognitive self-consciousness" scale (CSC) which is concerned with the extent to which individuals focus their attention on their own mental processes. Higher values in the former indicate a low confidence in cognitive abilities and higher values in the latter represent higher cognitive self-consciousness, which is seen as an indicator of metacognitive dysfunction similar to rumination and worry (Cartwright-Hatton \& Wells, 1997). Cronbach's $\alpha$ for both subscales was 0.8 , indicating high internal consistency (Howitt \& Cramer, 2005).

\section{Attention and Interference Performance}

The Color Word Interference Test (CWIT; Stroop, 1935) was used to record selective attention and executive functions. The test is divided into three parts: In the first part of the task, participants should read different words out loud as quickly as possible (WR). Subsequently, colors of different rectangles, which are presented on a screen, are to be named as fast as possible $(\mathrm{CN})$. In the third part, participants should name the printing color of a word that is incompatible with the meaning of the word (INT). The third part, the interference measure, allows to assess the ability to inhibit cognitive interference that occurs when the processing of a specific stimulus feature impedes the simultaneous processing of a second stimulus attribute. Participants have to suppress the automated tendency of semantic encoding in order to be able to carry out a controlled analysis of the printing ink incompatible with the meaning of the word. The test consists of three trials whereby in each trial the three parts are carried out in the order WR, CN and INT. A practice sheet is used beforehand to explain all three parts of the test to the participants using a sample line. After this practice sheet, subjects were informed about the preceding judgements of performance and confidence (see description below). In the present study mean completion times for each CWIT condition were recorded. The "Interference" score is calculated by subtracting the time needed to name the colored rectangles from the time needed to name the color of the words printed in incongruent colored ink (INT-CN). The mean Interference score over all three trials was build and used for analyses.

Groups were compared with regard to the performance in the CWIT to rule out that the effects of interest emerge from pure performance differences. Groups did not differ significantly in their performance regarding WR, CN and INT (for CWIT performance means see Fig. 1).

\section{Judgements of Performance and Confidence}

Participants' ability to monitor their own performance in the CWIT was assessed with judgements of performance (JOP) before their actual performance of every part of the task. Participants were asked to estimate the time they will need to perform the task (completion time in seconds). As an index of metacognitive monitoring ability $\left(\mathrm{Meta}_{\mathrm{acc}}\right), \mathrm{Meta}_{\mathrm{acc}}$ scores were calculated as the difference between judged and real performance time as recommended by Mengelkamp and Bannert (2009). This index can be used as measure for the assessment judgement accuracy: smaller differences indicate higher accuracies and, higher metacognitive monitoring abilities, respectively. In order to ensure that the judgements are based on internal monitoring processes, participants did not receive any feedback about the actual performance. For further details on this method, see Kessel et al. (2014). Furthermore, judgements of confidence (JOC) were assessed, whereby participants had to indicate, after each judgement, how confident they were that their time judgements are correct on a 6-point Likert scale ranging from 0 to $100 \%$ (Fig. 1 illustrates the respective JOP, the actual performance and the JOC separated for each part of the CWIT). For the main analysis JOPs, JOC and Meta $\mathrm{acc}_{\text {ac }}$ means were calculated over all three trials and parts.

\section{Statistical Analyses}

Data were analysed using IBM SPSS Statistics 25. Adequate sample size was calculated with G*Power 3.1 (Faul, 
Fig. 1 Mean performance of the WR, CN and INT parts of the CWIT and respective judgements

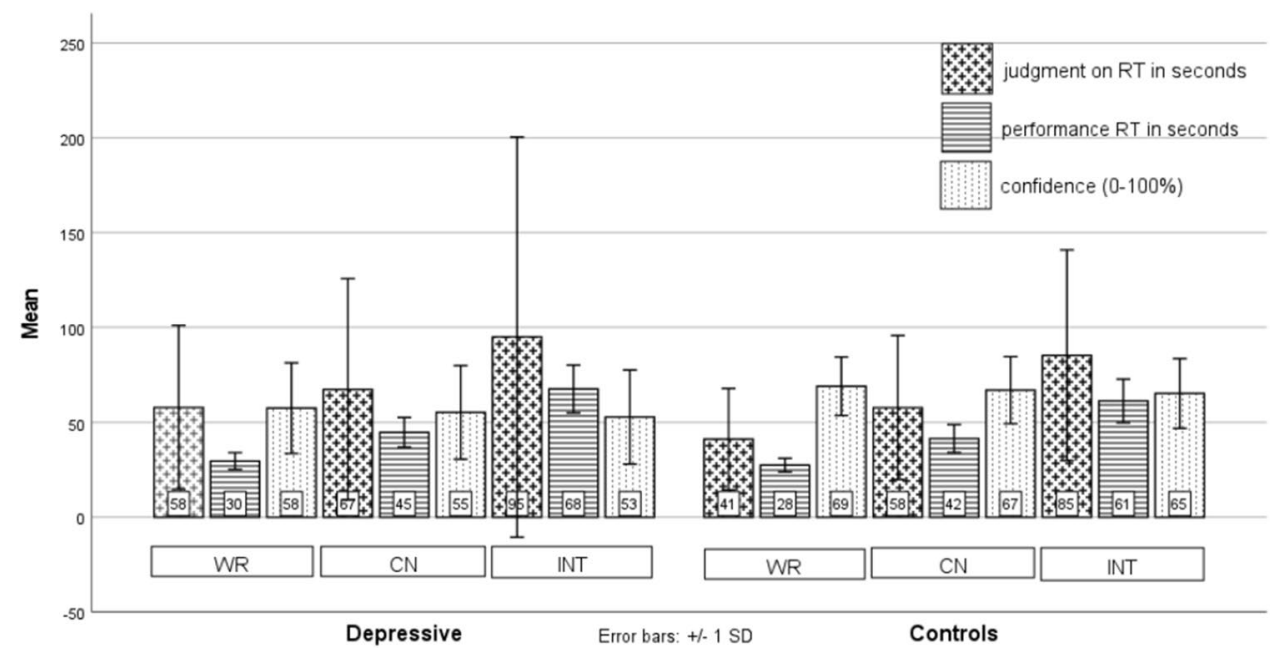

Erdfelder, Lang, \& Buchner, 2007) conducting a power analyses for $\mathrm{F}$ tests/MANOVA with repeated measures, between factors including the parameters: Effect size $\mathrm{f}=0.25 ; \alpha=$ 0.05 ; power $=0.90$; number of groups $=2$; number of measurements $=36$; correlations among repeated measures $=$ 0.3 . This a priori analysis revealed an adequate sample size of $\mathrm{N}=56$.

\section{Group Differences in Cognitive and Metacognitive Performance}

A between subjects Multiple Analysis of Variance (MANOVA) was used to compare the groups' means of interest which were the mean scores in the questionnaire measures DESC, EQ_DP, EQ_ASP, DFS_F, DFS_D, IS_VI, IS_MLI, MKF_CC and MKF_CSC and the mean performances with regard to the CWIT which were the mean Interference performance (INT-CN), the mean $\mathrm{Meta}_{\mathrm{acc}}$ and mean JOC. In the case of a significant MANOVA finding, post-hoc tests of between-subjects effects, partial eta squared effect sizes $\left(\eta_{p}{ }^{2}\right.$; Cohen, 1988) and standardized effect sizes (ES) with the respective confidence intervals (CI, Hedges bias corrected) are reported for each comparison.

\section{Associations Between Depression Severity, Decentering, Aspects of Self-conscious Attention and Individual Estimations with Regard to Performance and Confidence}

To investigate in how far depressiveness, metacognitive abilities and confidence are associated two-tailed Pearson correlation analyses were calculated for the questionnaire data (DESC, EQ_DP, EQ_ASP, DFS_F, DFS_D, IS_VI, IS_MLI, MKF_CC and MKF_CSC) as well as the mean Meta ${ }_{\mathrm{acc}}$ and mean JOC considering the associations with regard to the total sample $(N=60)$. Results are reported on a significance level of $p<.05$. According to Cohen's guidelines (1988),
Pearson's correlations of $r=.1$ represent a small, $r=.3$ a medium, and $r=.5$, a large effect.

\section{Regression}

Finally, to investigate in how far the derived correlates predict depression a blockwise linear regression was conducted for the total sample $(\mathrm{N}=60)$ introducing the DESC score as dependent variable. According to the results from the correlation analyses, the order of entry of the independent variables was determined by strength of the association with the DESC score. To predict depression (DESC), first the EQ factors (EQ_ASP, EQ_DP) were entered stepwise into the analysis, in the next stages DFS (DFS_D, DFS_F), MKF (MKF_CC, MKF_CSC) and ISI (ISI_VI, ISI_MLI) factors were entered stepwise respectively. In this hierarchical approach, the incremental predictive power of the (competing) predictors was determined.

\section{Results}

The MANOVA comparing the groups' questionnaire scores, as well as the Interference score of the CWIT and the Meta and JOCs revealed a significant main effect $(\mathrm{F}(12,47)=$ $17.37, p<.000, \eta_{p}{ }^{2}=.82$ ). Means and standard deviations of the groups and the tests of between-subjects effects for each dependent variable are displayed in Table 2 showing significant differences in all but three variables: First, the groups did not differ with respect to ISI_VI (Patients: $M_{I S I+V I}$ $=109.6, s d=14.2$; Control group: $M_{I S I_{-} V I}=114.9, s d=$ $9.4 ; \mathrm{F}(1,58)=2.91, p=.09)$, indicating that the groups have come to the similar self-assessments of their verbal intelligence. Second, participants actual Interference performances in the CWIT did not differ (Patients: $M_{\text {Interference }}$ $=22.9, s d=8.6$; Control group: $M_{\text {Interference }}=19.9, s d=$ 
Table 2 Means $(M)$, standard deviations $(s d)$ and betweensubject effects

\begin{tabular}{|c|c|c|c|c|c|c|c|c|c|}
\hline \multirow[b]{2}{*}{ Variables $^{\mathrm{a}}$} & \multicolumn{2}{|c|}{ Depressive } & \multicolumn{2}{|c|}{ Controls } & \multicolumn{5}{|c|}{ Group differences } \\
\hline & $M$ & $s d$ & $M$ & $s d$ & $F^{b}$ & $p$ & $\eta_{p}^{2}$ & $E S^{c}$ & $C I^{c}$ \\
\hline DESC & 20.5 & 6.3 & 4.1 & 3.3 & 159.6 & $<.001$ & .73 & 3.22 & $2.45-3.99$ \\
\hline EQ_DP & 1.2 & .5 & 2.3 & .7 & 50.5 & $<.001$ & .47 & -1.78 & $-2.38--1.19$ \\
\hline EQ_ASP & 1.8 & .7 & 3.0 & .5 & 64.6 & $<.001$ & .53 & -1.95 & $-2.56--1.33$ \\
\hline DFS_F & 2.8 & 0.5 & 3.6 & 0.6 & 32.4 & $<.001$ & .36 & -1.43 & $-2.00--.86$ \\
\hline DFS_D & 3.9 & 0.6 & 2.5 & 0.5 & 87.9 & $<.001$ & .60 & 2.50 & $1.83-3.18$ \\
\hline ISI_VI & 109.6 & 14.2 & 114.9 & 9.4 & 2.9 & .09 & .05 & -.43 & $-.95-.08$ \\
\hline ISI_MLI & 105.7 & 14.1 & 112.4 & 7.8 & 5.1 & $<.05$ & .08 & -.58 & $-1.10--.06$ \\
\hline MKF_CC & 14.3 & 4.7 & 10.2 & 3.4 & 15.3 & $<.001$ & .21 & .99 & $.45-1.52$ \\
\hline MKF_CSC & 16.4 & 4.1 & 11.3 & 3.4 & 26.9 & $<.001$ & .32 & 1.34 & $.78-1.90$ \\
\hline JOC & 55.3 & 23.8 & 67.2 & 16.2 & 5.1 & $<.05$ & .08 & -.58 & $-1.09--.06$ \\
\hline Meta $_{\mathrm{acc}}$ & 26.0 & 64.1 & 18.0 & 34.8 & .4 & .55 & .01 & .15 & $-.35-.66$ \\
\hline Interference & 22.9 & 8.6 & 20.0 & 7.0 & 2.2 & .15 & .04 & .37 & $-.15-.88$ \\
\hline
\end{tabular}

${ }^{\mathrm{a}}$ DESC $=$ Rasch-based Depression Screening; EQ_DP $=$ Experiences Questionnaire - Distanced Perspective Scale; EQ_ASP = Experiences Questionnaire - Accepting Self Perception Scale; DFS_F = Functional and Dysfunctional Self-consciousness questionnaire - Functional Scale; DFS_D = Functional and Dysfunctional Self-consciousness questionnaire - Dysfunctional Scale; ISI_VI = Inventar zur Selbsteingeschätzten Intelligenz - Verbal Intelligence Scale; ISI_MLI = Inventar zur Selbsteingeschätzten Intelligenz - Mathematical Logical Intelligence Scale; MKF_CC = Metacognitions Questionnaire - Cognitive Confidence Scale; MKF_CSC = Metacognitions Questionnaire - Cognitive Self Consciousness Scale; JOC $=$ mean confidence judgement; Meta $_{\mathrm{acc}}=$ mean metacognitive monitoring accuracy; Interference $=$ mean Interference score in the Color Word Interference Test. ${ }^{\mathrm{b}} \mathrm{F}$ statistic of the between-subjects effects revealed by the MANOVA. c: $\mathrm{ES}=$ Bias corrected (Hedges) standardized effect size; $\mathrm{CI}=$ confidence interval
7.0; $\mathrm{F}(1,58)=2.16, p=.15)$. And third, with respect to the Meta $_{\text {acc }}$, i.e. the mean differences between judged and real time performance, results reveal equally precise judgements of performance in the two groups (Patients: $M_{\text {Metaacc }}=26.0$, $s d=60.1 ;$ Control group: $M_{\text {Metaacc }}=18.0, s d=34.8 ; \mathrm{F}(1,58)$ $=0.37, p=.55)$. Interestingly, albeit similar performances and judgements thereof, groups significantly differed with regard to their perceived confidence in the judgements, in that patients with depressive episodes indicated an overall lower confidence in their judgements as compared to the control group (Patients: $M_{J O C}=55.3, s d=23.8$; Control group: $M_{J O C}=67.2, s d=16.2 ; \mathrm{F}(1,58)=5.1, p<.05 ; \eta_{p}{ }^{2}$ $=.08)(\mathrm{ES}:-.58$; CI: $-1.09--.06)$.
In line with these MANOVA findings, post-hoc analyses of variance comparing group differences regarding Meta $_{\text {acc }}$ and JOC separately for each single part (WR, CN, INT) of the CWIT, revealed a very similar picture, with no Meta $a_{\text {acc }}$ group differences but significant differences in the groups' confidence (see Table 3).

\section{Associations Between Variables}

The total samples Pearson's $r$ correlations between the variables are presented in Table 4. Here, it becomes evident that the extent of depressive symptoms, as acquired with the DESC, is negatively associated with rather "functional"
Table 3 Means $(M)$, standard deviations $(s d)$ and betweensubject effects regarding the three parts of the CWIT separately

\begin{tabular}{|c|c|c|c|c|c|c|c|c|c|}
\hline \multirow[b]{2}{*}{ Variables $^{\mathrm{a}}$} & \multicolumn{2}{|c|}{ Depressive } & \multicolumn{2}{|c|}{ Controls } & \multicolumn{5}{|c|}{ Group differences } \\
\hline & $M$ & $s d$ & $M$ & $s d$ & $\overline{F^{b}}$ & $p$ & $\eta_{p}{ }^{2}$ & $E S^{c}$ & $C I^{c}$ \\
\hline Meta $_{\text {acc }}$ Part 1 WR & 28.3 & 42.8 & 13.7 & 25.1 & 2.6 & .11 & .04 & .41 & $-.10-.92$ \\
\hline Meta $_{\mathrm{acc}}$ Part $2 \mathrm{CN}$ & 22.6 & 57.0 & 16.3 & 35.6 & .3 & .61 & .01 & .13 & $-.37-.64$ \\
\hline Meta $_{\text {acc }}$ Part 3 INT & 27.3 & 101.6 & 24.0 & 49.5 & .0 & .88 & .00 & .04 & $-.47-.55$ \\
\hline JOC Part 1 & 57.6 & 23.9 & 69.1 & 15.4 & 5.0 & $<.05$ & .08 & -.57 & $-1.08--.05$ \\
\hline JOC Part 2 & 55.3 & 24.7 & 67.1 & 17.7 & 4.5 & $<.05$ & .07 & -.54 & $-1.05--.02$ \\
\hline JOC Part 3 & 52.9 & 24.8 & 65.3 & 18.4 & 4.9 & $<.05$ & .08 & -.56 & $-1.08--.05$ \\
\hline
\end{tabular}

${ }^{\mathrm{a}} \mathrm{Meta}_{\mathrm{acc}}=$ mean metacognitive monitoring accuracy; $\mathrm{WR}=$ word reading; $\mathrm{CN}=$ color naming, $\mathrm{INT}=$ interference; Meta ${ }_{\text {acc }}$ Part 1: Diff (mean JOP WR - mean RT WR); Meta ${ }_{\text {acc }}$ Part 2: Diff (mean JOP CN mean RT CN); Meta $_{a c}$ c Part 3: Diff (mean JOP INT - mean RT INT); JOC = judgement of confidence; $\mathrm{JOP}=$ judgement of performance; ${ }^{\mathrm{b}} \mathrm{F}$ statistic of the between-subjects effects revealed by the ANOVAs. $\mathrm{c}$ : $\mathrm{ES}=$ Bias corrected (Hedges) standardized effect size; $\mathrm{CI}=$ confidence interval 
Table 4 Correlation matrix between the variables considering the total sample $(N=60)$

\begin{tabular}{|c|c|c|c|c|c|c|c|c|c|c|}
\hline Variables $^{\mathrm{a}}$ & EQ_DP & EQ_ASP & DFS_F & DFS_D & ISI_VI & ISI_MLI & MKF_CC & MKF_CSC & JOC & Meta $_{\mathrm{acc}}$ \\
\hline DESC & $-.70 * *$ & $-.77 * *$ & $-.67 * *$ & $.77 * *$ & $-.43 * *$ & $-.40 * *$ & $.53 * *$ & $.47 * *$ & $-.43 * *$ & .20 \\
\hline EQ_DP & 1 & $.73 * *$ & $.55 * *$ & $-.78 * *$ & $.28^{*}$ & $.33 *$ & $-.52 * *$ & $-.51 * *$ & .21 & .05 \\
\hline EQ_ASP & & 1 & $.53 * *$ & $-.70 * *$ & $.35^{* *}$ & $.26^{*}$ & $-.44 * *$ & $-.35 * *$ & $.45^{* *}$ & -.06 \\
\hline DFS_F & & & 1 & $-.73 * *$ & $.35^{* *}$ & $.29 *$ & $-.49 * *$ & $-.41 * *$ & $.26^{*}$ & -.19 \\
\hline DFS_D & & & & 1 & $-.32 *$ & $-.29 *$ & $.61 * *$ & $.69 * *$ & $-.26^{*}$ & .05 \\
\hline ISI_VI & & & & & 1 & $.42 * *$ & $-.40 * *$ & -0.13 & $.35^{* *}$ & -.12 \\
\hline ISI_MLI & & & & & & 1 & $-.44 * *$ & $-.33 * *$ & .20 & -.18 \\
\hline MKF_CC & & & & & & & 1 & $.49 * *$ & -.19 & .18 \\
\hline MKF_CSC & & & & & & & & 1 & -.05 & .03 \\
\hline JOC & & & & & & & & & 1 & -.21 \\
\hline Meta $_{\mathrm{acc}}$ & & & & & & & & & & 1 \\
\hline
\end{tabular}

Notes: ** Correlation is significant at the 0.01 level (2-tailed). * Correlation is significant at the 0.05 level (2-tailed), Positive associations are highlighted in lighter grey, negative associations in darker grey; ${ }^{a}$ DESC $=$ Rasch-based Depression Screening; EQ_DP = Experiences Questionnaire - Distanced Perspective Scale; EQ_ASP = Experiences Questionnaire - Accepting Self Perception Scale; DFS_F = Functional and Dysfunctional Self-consciousness questionnaire - Functional Scale; DFS_D = Functional and Dysfunctional Self-consciousness questionnaire - Dysfunctional Scale; ISI_VI = Inventar zur Selbsteingeschätzten Intelligenz - Verbal Intelligence Scale; ISI_MLI = Inventar zur Selbsteingeschätzten Intelligenz - Mathematical Logical Intelligence Scale; MKF_CC = Metacognitions Questionnaire - Cognitive Confidence Scale;

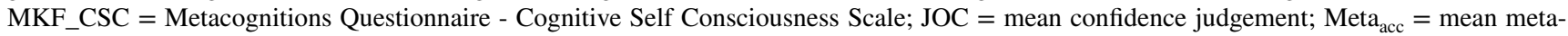
cognitive monitoring accuracy.

abilities, i.e. decentering, flexible self-attention, JOCs and self-assessed height of verbal and mathematical logical intelligence (EQ_DP, EQ_ASP, DFS_F, ISI_VI, ISI_MLI), whereas the extent of depressive symptoms showed positive associations with dysfunctional self-consciousness and low cognitive confidence (DFS_D, MKF_CC, MKF_CSC). Likewise, the different instruments reveal positive intercorrelations between scales representing functional and between scales representing dysfunctional characteristics, respectively, whilst negative correlations prevail for opposing measures.

\section{Regression Analysis}

Decentering (EQ_ASP, EQ_DP), self-conscious attention (DFS_D, DFS_F) and individual estimations with regard to cognitive performance (MKF_CC, MKF_CSC and ISI_ VI, ISI_MLI) were used in a stepwise multiple regression analysis to predict depressiveness (DESC). The correlations of the variables are shown in Table 4. As can be seen all correlations except for the one between MKF_CSC and ISI_VI $(\mathrm{p}=.16)$ were statistically significant $(\mathrm{p}<.05)$.

The prediction model contained four of the eight predictors and was reached in four steps with no variables removed. The model was statistically significant, $F(4,55)=$ $35.37, \mathrm{p}<.001$, and accounted for approximately $70 \%$ of the variance of depressiveness $\left(\mathrm{R}^{2}=.72\right.$, Adjusted $\left.\mathrm{R}^{2}=.70\right)$. Higher levels of DFS_D and lower levels of EQ_ASP, and to a lesser extent by lower levels of ISI_MLI and higher levels of EQ_DP primarily predicted depressiveness. The raw and standardized regression coefficients of the predictors together with their correlations with depressiveness, their squared semi-partial correlations, and their structure coefficients are shown in Table 5. Dysfunctional self-conscious attention (DFS_D) and the decentering factor "Accepting Self-Perception" (EQ_ASP) received the strongest weights
Table 5 Results of the stepwise regression analysis

\begin{tabular}{lllllll}
\hline Model & $\mathrm{b}$ & SE-b & Beta & Pearson $r$ & $\mathrm{Sr}^{2}$ & $\begin{array}{l}\text { Structure } \\
\text { coeffi- } \\
\text { cient }\end{array}$ \\
\hline Constant & 24.24 & 9.33 & & & & \\
EQ_ASP* & -4.75 & 1.22 & -.43 & -.77 & .08 & -.91 \\
EQ_DP & .023 & 1.50 & .002 & -.70 & .00 & -.82 \\
DFS_D* & 4.50 & 1.27 & .43 & .77 & .07 & .91 \\
ISI_MLI* & -.14 & .06 & -.17 & -.40 & .03 & -.47 \\
\hline
\end{tabular}

The dependent variable was depressiveness (DESC). $\mathrm{R}^{2}=.72$, Adjusted $\mathrm{R}^{2}=.70 ; \mathrm{Sr}^{2}$ is the squared semipartial correlation; *p $<.05$. 
in the model followed by self-assessed mathematical-logical intelligence (ISI_MLI); The decentering factor "Distanced Perspective" (EQ_DP) received the lowest of the four weights. With the sizeable correlations between the predictors, the unique variance explained by each of the variables indexed by the squared semi-partial correlations, was relatively low: decentering "Accepting Self- Perception" (EQ_ASP), dysfunctional self-conscious attention (DFS_D), self-assessed mathematical logical intelligence (ISI_MLI) and decentering "Distanced Perspective" uniquely accounted for approximately $8 \%, 7 \%, 3 \%$, and less than $1 \%$ of the variance of depressiveness. Inspection of the structure coefficients suggests that EQ_ASP and DFS_D were very strong indicators, EQ_DP was a strong indicator, and ISI_MLI was a moderate indicator of depressiveness.

\section{Discussion}

The present study aimed at investigating the association between cognitive and metacognitive functioning in depressive patients and healthy controls. On theoretical level, cognitive confidence has been linked to depression (Wells, 2008), and further, recent studies suspected a link between confidence judgements and depressiveness for healthy or subclinical populations (Moses-Payne et al., 2019; Rouault et al., 2018). However, especially in clinical samples there was relatively little empirical evidence for this so far. A review by Hoven et al. (2019) summarizes confidence abnormalities in subclinical and clinical symptomatology regarding, besides depression, different mental disorders. The review shows that in relation to studies of schizophrenia and obsessive-compulsive disorders, clinical studies of affective disorders, especially depression, are relatively sparse and predominantly investigate confidence regarding the domain of general knowledge and memory. Our research attempted to close this gap by investigating dysfunctional metacognition and confidence abnormalities regarding aspects of attention and cognitive flexibility in clinically depressed individuals.

Regarding cognitive functioning results revealed no significant difference between patients and aged-matched healthy controls in cognitive performance as measured with the CWIT. This indicates an equally well cognitive functioning in both groups, a finding, that differs from previous studies that report cognitive deficits in patients with depression (Gauggel \& Rathgeber, 2002; Marazziti et al., 2010; Marvel \& Paradiso, 2004; Mc Clintock et al., 2010; Vasic et al., 2007). Regarding the performance in the CWIT, a meta-analysis by Veiel (1997) found depressive patients to be impaired. In contrast to Veiel (1997) whom analysed studies with patients suffering from severe depression, most of our participants suffered from a mild or moderate episode, which could explain the diverging results. Veiel (1997) also reported a great variability of the findings, which might indicate that other factors, as for example metacognitive processes, may be of importance.

Similar to the findings regarding the performance in the CWIT, also the metacognitive judgements of performance, i.e. the difference between judged and real time performance, revealed as precise judgements in patients suffering from depression as compared to controls. This argues against the hypothesis of 'depressive realism' (Alloy \& Abramson, 1988) and supports some studies' findings (e.g. Dunn et al., 2007; Fu et al., 2005; Kalska et al., 1999). Similar to our finding of an enhanced dysfunctional self-focused attention in the patients, Dunn et al. (2007) found an elevated selffocused attention and a reduced positivity bias relative to healthy controls while the accuracy of the self-judgement was preserved in the dysphoric and depressed groups. At this point it is important to mention, that although over all performances did not differ between groups in the study at hand the design and the specific task looking at three different parts with increasing difficulty make it difficult to control for trial by trial learning or completely exclude or disentangle the influence of the participants general ability to estimate time.

Interestingly however, our study results further reveal, that the groups significantly differed with regard to their confidence in the judgements. Patients suffering from depressive episodes showed a lower overall confidence in their judgements compared to the individuals of the control group. This means the cognitive and metacognitive performance in the task was nearly the same for both groups but patients were less confident in their judgements. This is in line with the findings reported for foremost subclinical but also some clinical populations showing depressive symptomatology is going along with a comparably lower confidence in performance (Fu et al., 2005, 2012; Hoven et al., 2019; MosesPayne et al., 2019; Rouault et al., 2018). However, it must be mentioned at this point that by investigating a slightly different domain compared to the other studies on metacognition and confidence, some of the previously reported metacognitive measures of accuracy could not be calculated likewise and were therefore adapted to some extent.

Furthermore, the results at hand reveal evidence for the view of human metacognition as a quite basal cognitive ability involved in domain-unspecific judgement which is important for healthy functioning. Post-decisional judgements have been shown to provide useful information in optimizing control of behavior via parametric estimates and help decision making and evaluation. This in turn can lead to changes in mind or support ratings of confidence in an initial decision (for review see Yeung \& Summerfield, 2012). However, the confidence judgements in the present study were prospective, i.e. pre-decisional and hence were 
rather built on personal believes about owned abilities in the test domain. While an overconfidence across domains and transcending time is assumed in the healthy individual, we assumed an overall dysfunctional confidence reflected in lower overall ratings in the individuals suffering from depression. A study by Fleming et al. (2016) highlighted the importance of prospective judgements by comparing retroto prospective judgements in a healthy sample. The authors reported dissociable effects: retrospective judgements were strongly influenced by the speed and accuracy of the preceding decision, whereas prospective judgements were dependent on past confidence extending back over a longer time window. Further, global levels of confidence were correlated across judgements, which supports the assumption of a domain-general overconfidence in a "healthy" performance. Our research focused on the link between prospective judgements and an assumed lower confidence in performance in a sample suffering from depression. It has to be noted that the design differences prevent investigating the same scores compared to Fleming et al. (2016) and therefore results cannot be generalized or inferred to the findings in our depressed sample. Nevertheless, taking into account Flemings' findings, we suggest that the results of the present study rather reflect a trait-like self-fulfilling prophecy effect subsuming the patients' dysfunction regarding a longer period of time, than the sequential dependencies of confidence ratings, as found for example in feedback studies.

The lack of general confidence in their cognitive abilities found in the patients' judgements is further mirrored in what participants indicated regarding the different metacognitive abilities in the questionnaire assessments: participants with depression seemed to have less trust in their own abilities. Patients suffering from depression reported a lower confidence as compared to healthy controls as measured with the Cognitive Confidence subscale of the Metacognitive Questionnaire (MKF). Also in the other subscale of the MKF, Cognitive Self-Consciousness, significant higher scores indicating a metacognitive dysfunction was found in the patients. Further, patients suffering from depression reported significantly lower decentering abilities in both subscales of the decentering questionnaire which indicates that healthy individuals seemed better able to achieve a reflective and observing perspective on their own mental processes and that they were more able to experience themselves as a person with own characteristics. Here, results are in line with the findings reported by Teasdale et al. (2002) showing lower decentering abilities in depressive patients compared to healthy controls. Regarding self-assessed intelligence depressed individuals referred significant lower levels of verbal and mathematicallogical abilities to themselves compared to healthy controls, although groups actual intelligence as estimated with the WST did not differ. A relationship between well-being and self-assessed intelligence has already been demonstrated in narcissists (Zajenkowski \& Czarna, 2015). According to the extended agency model of narcissism (Campbell \& Foster, 2007), intelligence is a crucial agentic quality, and, estimating one's own IQ as low constitutes a failure of the self-regulatory system as it might also be the case in the present sample of depressed patients. The patients in the current sample also reported significant more dysfunctional attention abilities as measured with the DFS. This is in line with the notion that depressed individuals tended to allocate their attention inward towards themselves, their thoughts, and their feelings. Already in 1985, Smith, Ingram and Roth found self-focused attention to be correlated with depression and a negative evaluation of the self. In line with this, the closer inspection of the associations between clinical, cognitive and metacognitive measures revealed negative associations between depression severity and functional metacognitive abilities like decentering, flexible self-attention, high JOCs and self-assessed height of verbal and mathematical logical intelligence, and, positive associations with dysfunctional aspects as dysfunctional self-attention, and low cognitive confidence and self-consciousness. Similarly, the results of the regression analysis revealed that high scores in dysfunctional self-attention and lower scores in the decentering factor 'accepting self-perception' were the best predictors for depressiveness. Individuals with high scores in dysfunctional self-attention are thought to be in an inflexible or prolonged state of self-focused attention. The finding that decentering is diminished in depressive patients is in line with previous studies that found decentering to be a process promoting wellbeing. It seems that basal cognitive processes play a minor role for decentering abilities whereas affective components as aspects of self-acceptance and confidence seem to play an important role for the ability to decenter.

The present study makes an important contribution in bridging different aspects of metacognition to different aspects of attention. Taken together and in line with the findings regarding impacted (meta-)memory by Cipolli et al. (1996), the present studies' data point to further metacognitive dysfunction regarding aspects of (meta-)cognitive attention in patients with depression. All in all, our results further underline that patients make equally precise metacognitive judgements in the domain of attention but are less confident in comparison to healthy controls. Furthermore, the aspect of a lowered confidence in (meta-)cognitive abilities was further mirrored in the associations between metacognitive abilities, like decentering, aspects of self-conscious attention, self-assessed intelligence, metacognitive beliefs, judgements and monitoring tendencies and depressiveness.

Metacognitive processes play a vital role in the course and successful treatment of mental disorders. Papageorgiou and Wells (2003) showed that in depressive patients the contents of metacognitive beliefs have an influence on the rumination tendency and the maintenance of depressive 
symptoms. This finding fits the approach of classical cognitive behavioral therapy (CBT), which has primarily aimed at changing the content of dysfunctional cognitions (Beck, 2005). The effectiveness has been proven in numerous studies over the past decades. However, studies have shown that not only the change in cognitive content but also the change in the metacognitive perspective from which cognitions are viewed has a positive effect on the success of therapy and the likelihood of relapse (Corcoran \& Segal, 2008; Teasdale et al., 2002). Therefore, it seems to be important to have a closer look on the results of metacognitive assessments of attention in the study at hand. Future research should address these aspects in order to improve the treatment of patients with affective disorders.

Data availability Statement The datasets generated during and/or analysed during the current study are available from the corresponding author on reasonable request.

Author contributions Barbara Drueke, Thomas Forkmann, Siegfried Gauggel and Verena Mainz contributed to the study conception and design. Material preparation, data collection and analysis were performed by Barbara Drueke, Thomas Forkmann, Verena Mainz and Lorenz Weise. The first draft of the manuscript was written by Barbara Drueke and Verena Mainz and all authors commented on previous versions of the manuscript. All authors read and approved the final manuscript.

Funding Open Access funding enabled and organized by Projekt DEAL. START-program of the Faculty of Medicine, RWTH Aachen University (691201)

\section{Declarations}

Ethics approval The study has been approved by the local ethics committee (EK148/11) and has therefore been performed in accordance with the ethical standards laid down in the 1964 Declaration of Helsinki and its later amendments.

Consent to participate All persons gave their informed consent prior to their inclusion in the study. Details that might disclose the identity of the subjects under study were omitted.

Conflicts of interest The authors have no relevant financial or nonfinancial interests to disclose.

Ethical standards The study has been approved by the local ethics committee (EK148/11) and has therefore been performed in accordance with the ethical standards laid down in the 1964 Declaration of Helsinki and its later amendments. All persons gave their informed consent prior to their inclusion in the study. Details that might disclose the identity of the subjects under study were omitted.

Open Access This article is licensed under a Creative Commons Attribution 4.0 International License, which permits use, sharing, adaptation, distribution and reproduction in any medium or format, as long as you give appropriate credit to the original author(s) and the source, provide a link to the Creative Commons licence, and indicate if changes were made. The images or other third party material in this article are included in the article's Creative Commons licence, unless indicated otherwise in a credit line to the material. If material is not included in the article's Creative Commons licence and your intended use is not permitted by statutory regulation or exceeds the permitted use, you will need to obtain permission directly from the copyright holder. To view a copy of this licence, visit http://creativecommons.org/licenses/by/4.0/.

\section{References}

Alloy, L., \& Abrahamson, L. (1988). Depressive realism. Cognitive processes in depression, 441-85.

Aldahadha, B. (2021). Metacognition, mindfulness attention awareness, and their relationships with depression and anxiety. Journal of Rational-Emotive \& Cognitive-Behavior Therapy, 39(2), 183-200. https://doi.org/10.1007/s10942-020-00367-y

Arndt, A., Patzelt, J., Andor, T., Hoyer, J., \& Gerlach, A. L. (2011). Psychometrische Gütekriterien des Metakognitionsfragebogens (Kurzversion, MKF-30). Zeitschrift für Klinische Psychologie und Psychotherapie, 40, 107-114. https://doi.org/10.1026/1616-3443/ a000087

Beck, A. T. (2005). The current state of cognitive therapy: A 40-year retrospective. Archives of General Psychiatry, 62(9), 953-959.

Bernstein, A., Hadash, Y., Lichtash, Y., Tanay, G., Shepherd, K., \& Fresco, D. M. (2015). Decentering and related constructs: A critical review and metacognitive processes model. Perspectives on Psychological Science, 10(5), 599-617. https://doi.org/10.1177/ 1745691615594577

Borkowski, J. G. (1985). Signs of intelligence: Strategy generalization and metacognition. Academic.

Campbell, W. K., \& Foster, J. D. (2007). The narcissistic self: Background, an extended agency model, and ongoing controversies. The self, 115-138.

Carmody, J., Baer, R. A., Lykins, E. L. B., \& Olendzki, N. (2009). An empirical study of the mechanisms of mindfulness in a mindfulness-based stress reduction program. Journal of Clinical Psychology, 65(6), 613-626. https://doi.org/10.1002/jclp.20579

Cartwright-Hatton, S., \& Wells, A. (1997). Beliefs about worry and intrusions: The Meta-Cognitions Questionnaire and its correlates. Journal of anxiety disorders, 11(3), 279-296. https://doi.org/10. 1016/S0887-6185(97)00011-X

Cipolli, C., Neri, M., De Vreese, L. P., Pinelli, M., Rubichi, S., \& Lalla, M. (1996). The influence of depression on memory and metamemory in the elderly. Archives of Gerontology and Geriatrics, 23(2), 111-127. https://doi.org/10.1016/0167-4943(96)00712-1

Coelho, H. F., Canter, P. H., \& Ernst, E. (2007). Mindfulness-based cognitive therapy: Evaluating current evidence and informing future research. Journal of Consulting and Clinical Psychology, 75(6), 1000-1005. https://doi.org/10.1037/2326-5523.1.S.97

Cohen, J. (1988). Statistical power analysis for the behavioral sciences. Erlbaum.

Corcoran, K. M., \& Segal, Z. V. (2008). Metacognition in depressive and anxiety disorders: current directions. International Journal of Cognitive Therapy, 1(1), 33-44. https://doi.org/10.1521/ijct. 2008.1.1.33

Culot, C., Fantini-Hauwel, C., \& Gevers, W. (2021). The influence of sad mood induction on task performance and metacognition. Quarterly Journal of Experimental Psychology, 17470218211004205

Dunn, B. D., Dalgleish, T., Lawrence, A. D., \& Ogilvie, A. D. (2007). The accuracy of self-monitoring and its relationship to selffocused attention in dysphoria and clinical depression. Journal of Abnormal Psychology, 116(1), 1.

Ehrlinger, J., \& Dunning, D. (2003). How chronic self-views influence (and potentially mislead) estimates of performance. Journal of 
Personality and Social Psychology, 84(1), 5-17. https://doi.org/ 10.1037/0022-3514.84.1.5

Faul, F., Erdfelder, E., Lang, A. G., \& Buchner, A. (2007). G* Power 3: A flexible statistical power analysis program for the social, behavioral, and biomedical sciences. Behavior research methods, 39(2), 175-191. https://doi.org/10.3758/BF03193146

Flavell, J. H. (1979). Metacognition and cognitive monitoring: A new area of cognitive-developmental inquiry. American Psychologist, 34(10), 906-911. https://doi.org/10.1037/0003-066X.34.10.906

Fleming, S. M., Massoni, S., Gajdos, T. \& Vergnaud, J.-C. (2016). Metacognition abou the past and the future:quantifying common and distinct influences on prospective and retrospective judgments of self-perfomance. Neuroscience of Consciousness, 1-12

Forkmann, T., Boecker, M., Wirtz, M., Eberle, N., Westhofen, M., Schauerte, P., et al. (2009). Development and validation of the Rasch-based depression screening (DESC) using Rasch analysis and structural equation modelling. Journal of Behavior Therapy and Experimental Psychiatry, 40, 468-478. https://doi.org/10. 1016/j.jbtep.2009.06.003

Forkmann, T., Boecker, M., Wirtz, M., Glaesmer, H., Brahler, E., Norra, C., et al. (2010). Validation of the Rasch-based Depression Screening in a large scale German general population sample. Health \& Quality of Life Outcomes, 8, 105. https://doi.org/ 10.1186/1477-7525-8-105

Fresco, D. M., Moore, M. T., van Dulmen, M. H. M., Segal, Z. V., Ma, S. H., Teasdale, J. D., \& Williams, J. M. G. (2007). Initial psychometric properties of the experiences questionnaire: validation of a self-report measure of decentering. Behavior Therapy, 38, 234-246. https://doi.org/10.1016/j.beth.2006.08.003

Fu, T., Koutstaal, W., Fu, C. H., Poon, L., \& Cleare, A. J. (2005). Depression, confidence, and decision: Evidence against depressive realism. Journal of Psychopathology and Behavioral Assessment, 27(4), 243-252.

Fu, T. S. T., Koutstaal, W., Poon, L., \& Cleare, A. J. (2012). Confidence judgement in depression and dysphoria: The depressive realism vs. negativity hypotheses. Journal of Behavior Therapy and Experimental Psychiatry, 43, 699-704. https://doi.org/10. 1016/j.jbtep.2011.09.014

Gauggel, S., \& Rathgeber, K. (2002). Neuropsychologie affektiver Störungen: Eine selektive Übersicht. Zeitschrift für Neuropsychologie, 13(4), 301-312.

Gecht, J., Kessel, R., Mainz, V., Gauggel, S., Drueke, B., Scherer, A., \& Forkmann, T. (2014). Measuring decentering in self-reports: Psychometric properties of the Experiences Questionnaire in a German sample. Psychotherapy Research, 24, 67-79. https:// doi.org/10.1080/10503307.2013.821635

Hargus, E., Crane, C., Barnhofer, T., \& Williams, J. M. G. (2010). Effects of mindfulness on meta-awareness and specificity of describing prodromal symptoms in suicidal depression. Emotion, 10(1), 34-42. https://doi.org/10.1037/a0016825

Hayes, S. C. (2004). Acceptance and commitment therapy, relational frame theory, and the third wave of behavioral and cognitive therapies. Behavior Therapy, 35(4), 639-665. https://doi.org/ 10.1016/S0005-7894(04)80013-3

Hiller, W., Zaudig, M., \& Mombour, W. (1996). IDCL: International Diagnostic Checklists for ICD-10 and DSM-IV. Hogrefe \& Huber Publishers.

Hoven, M., Lebreton, M., Engelmann, J. B., Denys, D., Luigjes, J., \& van Holst, R. J. (2019). Abnormalities of confidence in psychiatry: an overview and future perspectives. Translational psychiatry, 9(1), 1-18.

Hoyer, J. (2000). Questionnaire of Dysfunctional and Functional Self-Consciousness (DFS): Theoretical concept, reliability and validity. Diagnostica, 46, 140-148. https://doi.org/10.1026/ 0012-1924.46.3.140
Howitt, D., \& Cramer, D. (2005). Introduction to research methods in psychology. Pearson Education Limited.

Kalska, H., Punamaki, R. L., Makinen-Pelli, T., \& Saarinen, M. (1999). Memory and metamemory functioning among depressed patients. Applied Neuropsychology, 6(2), 96-107. https://doi. org/10.1207/s15324826an0602_5

Keren, G. (1991). Calibration and probability judgements: Conceptual and methodological issues. Acta Psychologica, 77(3), 217-273. https://doi.org/10.1016/0001-6918(91)90036-Y

Kessel, R., Gecht, J., Forkmann, T., Drueke, B., Gauggel, S., \& Mainz, V. (2014). Metacognitive monitoring of attention performance and its influencing factors. Psychological Research, 78(4), 597-607. https://doi.org/10.1007/s00426-013-0511-y

Koriat, A. (1997). Monitoring one's own knowledge during study: A cue-utilization approach to judgments of learning. Journal of Experimental Psychology: General, 126(4), 349-370. https:// doi.org/10.1037/0096-3445.126.4.349

Koriat, A. (2007). Metacognition and consciousness. In P. D. Zelazo, M. Moscovitch, \& E. Thompson (Eds.), Cambridge handbook of consciousness. Cambridge University Press.

Koriat, A., \& Helstrup, T. (2007). Metacognitive aspects of memory. In S. Magnussen \& T. Helstrup (Eds.), Everyday Memory. Psychology Press.

Koriat, A., \& Shitzer-Reichert, R. (2002). Metacognitive judgments and their accuracy. Springer. https://doi.org/10.1007/ 978-1-4615-1099-4_1

Kraft, B., Jonassen, R., Stiles, T. C., \& Landrø, N. (2017). Dysfunctional metacognitive beliefs are associated with decreased executive control. Frontiers in psychology, 8, 593. https://doi.org/10. 3389/fpsyg.2017.00593

Lebois, L. A. M., Papies, E. K., Gopinath, K., Cabanban, R., Quigley, K. S., Krishnamurthy, V., . . Barsalou, L. W. (2015). A shift in perspective: decentering through mindful attention to imagined stressful events. Neuropsychologia, 75, 505-524. https://doi.org/ 10.1016/j.neuropsychologia.2015.05.030

Marazziti, D., Consoli, G., Picchetti, M., Carlini, M., \& Faravelli, L. (2010). Cognitive impairment in major depression. European journal of pharmacology, 626(1), 83-86. https://doi.org/10. 1016/j.ejphar.2009.08.046

Marvel, C. L., \& Paradiso, S. (2004). Cognitive and neurological impairment in mood disorders. Psychiatric Clinics of North America, 27(1), 19-36. https://doi.org/10.1016/S0193-953X(03) 00106-0

McClelland, A. G., \& Bolger, F. (1994). The calibration of subjective probability: Theories and models 1980-94.

McClintock, S. M., Hussain, M., Greer, T. L., \& Cullum, C. M. (2010). Association between depressive severity and neurocognitive function in major depressive disorder: A review and synthesis. Neuropsychology, 24(1), 9-34. https://doi.org/10.1037/a0017336

Mengelkamp, C., \& Bannert, M. (2009). Judgements about knowledge: Searching for factors that influence their validity. Electronic Journal of Research in Educational Psychology, 7, 163-190.

Moses-Payne, M. E., Rollwage, M., Fleming, S. M., \& Roiser, J. P. (2019). Postdecision evidence integration and depressive symptoms. Frontiers in psychiatry, 10, 639.

Nelson, T. O. (1990). Metamemory: A theoretical framework and new findings. In Psychology of learning and motivation (Vol. 26, pp. 125-173). Academic Press. https://doi.org/10.1016/S00797421(08)60053-5

Ong, J. C., Ulmer, C. S., \& Manber, R. (2012). Improving sleep with mindfulness and acceptance: a metacognitive model of insomnia. Behaviour Research and Therapy, 50(11), 651-660. https://doi. org/10.1016/j.brat.2012.08.001

Papageorgiou, C., \& Wells, A. (2001). Metacognitive beliefs about rumination in recurrent major depression. Cognitive and 
Behavioral Practice, 8(2), 160-164. https://doi.org/10.1016/ S1077-7229(01)80021-3

Rammstedt, B., \& Rammsayer, T. (2002). Die Erfassung der selbsteingeschätzten Intelligenz: Konstruktion, teststatistische Überprüfung und erste Ergebnisse des Inventars zur selbsteingeschätzten Intelligenz (ISI). Zeitschrift für Differentielle und Diagnostische Psychologie, 23, 435-446. https://doi.org/10.1024/0170-1789. 23.4.435

Rouault, M., Seow, T., Gillan, C. M., \& Fleming, S. M. (2018). Psychiatric symptom dimensions are associated with dissociable shifts in metacognition but not task performance. Biological psychiatry, 84(6), 443-451.

Rock, P. L., Roiser, J. P., Riedel, W. J., \& Blackwell, A. D. (2014). Cognitive impairment in depression: a systematic review and metaanalysis. Psychological medicine, 44(10), 2029-2040. https://doi. org/10.1017/S0033291713002535

Safran, J. D., \& Segal, Z. V. (1990). Interpersonal process in cognitive therapy. Jason Aronson.

Schmidt, K. H., \& Metzler, P. (1992). Wortschatztest (WST). Beltz.

Sheppard, L. C., \& Teasdale, J. D. (2004). How does dysfunctional thinking decrease during recovery from major depression? Journal of Abnormal Psychology, 113(1), 64. https://doi.org/10.1037/ 0021-843X.113.1.64

Smith, T. W., Ingram, R. E., \& Roth, D. L. (1985). Self-focused attention and depression: Self-evaluation, affect, and life stress. Motivation and emotion, 9(4), 381-389. https://doi.org/10.1007/BF009 92207

Soderstrom, N. C., Davalos, D. B., \& Vázquez, S. M. (2011). Metacognition and depressive realism: Evidence for the level-of-depression account. Cognitive Neuropsychiatry, 16(5), 461-472. https:// doi.org/10.1080/13546805.2011.557921

Spada, M. M., Georgiou, G. A., \& Wells, A. (2010). The relationship among metacognitions, attentional control, and state anxiety. Cognitive behaviour therapy, 39(1), 64-71. https://doi.org/10.1080/ 16506070902991791

Sternberg, R. J. (1984). What should intelligence tests test? Implications for a triarchic theory of intelligence for intelligence testing. Educational Researcher, 13(1), 5-15. https://doi.org/10.3102/ 0013189X013001005

Stroop, J. R. (1935). Studies of interference in serial verbal reactions. Journal of Experimental Psychology, 18, 643-662. https://doi. org/10.1037/h0054651
Teasdale, J. D., Moore, R. G., Hayhurst, H., Pope, M., Williams, S., \& Segal, Z. V. (2002). Metacognitive awareness and prevention of relapse in depression: empirical evidence. Journal of Consulting and Clinical Psychology, 70(2), 275-287. https://doi.org/10.1037/ 0022-006X.70.2.275

Tournier, I., \& Postal, V. (2011). Effects of depressive symptoms and routinization on metamemory during adulthood. Archives of Gerontology and Geriatrics, 52(1), 46-53. https://doi.org/10.1016/j. archger.2010.01.019

Vasic, N., Wolf, R. C., \& Walter, H. (2007). Executive functions in patients with depression. The role of prefrontal activation. Der Nervenarzt, 78(6), 628-630. https://doi.org/10.1007/ s00115-006-2240-6

Wells, A. (2002). Emotional disorders and metacognition: Innovative cognitive therapy. John Wiley \& Sons.

Wells, A. (2008). Metacognitive therapy: Cognition applied to regulating cognition. Behavioural and cognitive psychotherapy, 36(6), 651-658. https://doi.org/10.1017/S1352465808004803

Wells, A., \& Cartwright-Hatton, S. (2004). A short form of the metacognitions questionnaire: properties of the MCQ-30. Behaviour Research and Therapy, 42, 385-396. https://doi.org/10.1016/ S0005-7967(03)00147-5

Wells, A., \& Matthews, G. (1994). Attention and emotion: A clinical perspective. Lawrence Erlbaum.

Wells, A., \& Matthews, G. (1996). Anxiety and cognition. Current Opinion in Psychiatry, 9(6), 422-426.

Yeung, N., \& Summerfield, C. (2012). Metacognition in human decision-making: confidence and error monitoring. Philosophical Transactions of the Royal Society B: Biological Sciences, 367(1594), 1310-1321. https://doi.org/10.1098/rstb.2011.0416

Zajenkowski, M., \& Czarna, A. Z. (2015). What makes narcissists unhappy? Subjectively assessed intelligence moderates the relationship between narcissism and psychological well-being. Personality and Individual Differences, 77, 50-54. https://doi.org/10. 1016/j.paid.2014.12.045

Publisher's Note Springer Nature remains neutral with regard to jurisdictional claims in published maps and institutional affiliations. 